\title{
Modeling of Turbine-Boiler Coordinated System Based on Online Support Vector Machine
}

\author{
Meihua $\mathrm{Li}^{1, \mathrm{a})}$, Changliang Liu ${ }^{2, \text { b) }}$ \\ ${ }^{1}$ North China Electric Power University, Baoding, Hebei 071000, China. \\ ${ }^{2}$ State Key Laboratory of Alternate Electrical Power System with Renewable Energy Sources, \\ North China Electric Power University, Beijing 102206, China. \\ a) Imhdwy1121@163.com \\ b)13603123513@163.com
}

\begin{abstract}
The simplified non-linear unit model not only can provide the inherent rules by control system design, but also can be use as a simulation process to evaluate the performance of the control algorithms. Using the nonlinear mapping characteristics of Online Support Vector Machine (Online SVM), the dynamic mathematical models of turbine-boiler coordinated system were established, and the simulation research were conducted. Operation data simulation shows that the model can perfectly reflect the dynamic characteristics of turbine-boiler coordinated system. When a new condition appeared in the process which did not include in the working condition data, the model can take advantage of the new data to realize online update, adding a new support vector, thus has a good reappearance.
\end{abstract}

Keywords: Modeling, Coordinated System, Online, Support Vector Machine

\section{INTRODUCTION}

At present, modeling of turbine-boiler coordinated system is mainly divided into mechanism modeling and test modeling. Because the power plant coordination system is very complex, it is very difficult to understand every detail. Therefore, the mechanism modeling has some limitations. In many cases, many factors should be ignored. Test modeling has the characteristics of black box, and it doesn't need to understand the internal mechanism of objects, so it has attracted the attention of technicians and scholars. In recent years, modeling methods for power plant coordination systems are based on neural network modeling and support vector regression modeling [1]. The basic support vector regression modeling is steady state model. When load changes are more frequent and coal quality and other complex factors are affected, steady state modeling is no longer able to reproduce well the operation of the field. Therefore, the following two points should be considered as follows: 1 ) from the point of view of control optimization, a model that can reflect the dynamic characteristics of the system can be built to optimize the dynamic process on this basis; 2) the model needs to have adaptive function to avoid the effect of model mismatch.

In this paper, based on the dynamic modeling method of online adaptive support vector machine, a mathematical model is established to reflect the characteristics of the coordination system of the furnace, and the accuracy and adaptability of the model are verified by using the data of the 600MW power plant in a power plant. First, the computation process of online support vector machine is introduced. Then, the accuracy, real-time and adaptive ability of the model are verified by the operation data of the unit.

\section{THE PRINCIPLE OF ONLINE SUPPORT VECTOR MACHINE}

\section{Introduction of Support Vector Machine}

Support vector machine modeling is a nonlinear model identification method based on the basic principle of structural risk minimization. It has the advantages of good generalization and not easy to fall into the local minima. 
For a given set of training data $T=\left\{x_{i}, y_{i}\right\}(i=1,2, \ldots, l), x_{i} \in R^{N}, y_{i} \in R$, Nonlinear mapping $\Phi$ is used to map data $x_{i}$ to high-dimensional feature space $F$. In this space, we find the mapping relationship $f$, which can estimate the given data well. According to the statistical learning theory, we construct the regression equation.

$$
\left\{\begin{array}{c}
f(x)=w \Phi(\mathrm{x})+\mathrm{b} \\
\Phi: R^{N} \rightarrow F \\
w \in F
\end{array}\right.
$$

The function estimation problem in Formula 1 is equivalent to the minimization of the following functions:

$$
\begin{aligned}
R_{\text {reg }}[f] & =R_{e m p}[f]+\lambda\|w\|^{2} \\
& =\sum_{i=1}^{t} C\left(e_{i}\right)+\lambda\|w\|^{2}
\end{aligned}
$$

The optimization problem can be obtained by the formula (2).

$$
\left\{\begin{array}{c}
\min D=\frac{1}{2}\|w\|^{2}+C \sum_{i=1}^{t}\left(\xi_{i}+\xi_{i}^{*}\right) \\
\text { s.t. } y_{i}-w^{T} x_{i}-b \leq \varepsilon+\xi_{i} \\
w^{T} x_{i}+b-y_{i} \leq \varepsilon+\xi_{i}{ }^{*} \\
\xi_{i} \geq 0 \\
\xi_{i}{ }^{*} \geq 0
\end{array}\right.
$$

By solving formula (3), we can get $w$ and $b$. The Lagrange equation of optimization problem (3) is constructed. The dual problem of this equation is

$$
\left\{\begin{array}{c}
\min W=\frac{1}{2} \sum_{i=1}^{t} K\left(x_{i}, x_{j}\right)\left(\alpha_{i}-\alpha_{i}^{*}\right)\left(\alpha_{j}-\alpha_{j}^{*}\right)+ \\
\varepsilon \sum_{i=1}^{t}\left(\alpha_{i}+\alpha_{i}^{*}\right)-\sum_{i=1}^{t} y_{i}\left(\alpha_{i}-\alpha_{i}^{*}\right) \\
\text { s.t. } 0 \leq \alpha_{i}, \alpha_{i}^{*} \leq C_{i}, i=1,2, \ldots, l \\
\sum_{i=1}^{t}\left(\alpha_{i}-\alpha_{i}^{*}\right)=0
\end{array}\right.
$$

Where: $\alpha_{i}$ and $\alpha_{i}^{*}$ are Lagrange multipliers, $K\left(x_{i}, x_{j}\right)=\Phi\left(x_{i}\right) \Phi\left(x_{j}\right)$. is kernel function. Therefore, a regression equation can be constructed.

$$
f(x)=\sum_{i=1}^{t}\left(\alpha_{i}-\alpha_{i}^{*}\right) K\left(x_{i}, x_{j}\right)+b
$$

Through Lagrange multiplier and Karush-Kuhn-Tucker (KKT) conditions, the training data set can be divided into 3 sets: error support vector set [2]: $E=\left\{i|| \theta_{i}|=C| h,\left(x_{i}\right) \mid>\varepsilon\right\}$; boundary support vector set: $S=$ $\left\{i|0 \leq| \theta_{i}|\leq C| h,\left(x_{i}\right) \mid=\varepsilon\right\}$; residual sample set: $R=\left\{i|| \theta_{i}|=0| h,\left(x_{i}\right) \mid<\varepsilon\right\}$. Among them, $\theta_{i}=\alpha_{i}-\alpha_{i}^{*}$, $h\left(x_{i}\right)=f\left(x_{i}\right)-y_{i}$.

\section{The Calculation Process of Online Support Vector Machine}

The main step of online support vector machine algorithm is, when adding a sample $\left(x_{c}, y_{c}\right)$ to training set, we first judge whether the change $\Delta \theta_{c}$ is positive or negative [3,4]. That is:

$$
\operatorname{sign}\left(\Delta \theta_{c}\right)=\operatorname{sign}\left(-h\left(x_{c}\right)\right)
$$

Next, we should decide the boundary of the maximum change value of $\Delta \theta_{c}$ according to every sample in the training set $T$. In order to simplify the derivation process. We only consider the case of $\Delta \theta_{c}>0$, and the case of $\Delta \theta_{c}<0$ is similar.

For the new sample, there are two kinds of processing:

Case 1 : If $h\left(x_{c}\right)$ is changed from $h\left(x_{c}\right)<\varepsilon$ to $h\left(x_{c}\right)=\varepsilon$, the new sample $x_{c}$ is added to the set $S$, and the algorithm is terminated.

Case 2: If $\Delta \theta_{c}$ is changed from $\Delta \theta_{c}<C$ to $\Delta \theta_{c}=C$, the new sample $x_{c}$ is added to the set $E$, and the algorithm is terminated.

For each sample in set $S$, we do the following processing:

Case 3 : If $\theta_{i}$ is increased from $0<\left|\theta_{i}\right|<C$ to $\left|\theta_{i}\right|=C$, the sample $x_{i}$ is changed from set $S$ to set $E$. If $\theta_{i}$ changes to $\left|\theta_{i}\right|=0$, the sample $x_{i}$ is changed from set $S$ to set $R$. 
For each sample in set $E$, we do the following processing:

Case 4 : If $h\left(x_{i}\right)$ is changed from $h\left(x_{i}\right)>\varepsilon$ to $h\left(x_{i}\right)=\varepsilon$, the sample $x_{i}$ is changed from set $E$ to set $S$.

For each sample in set $R$, we do the following processing:

Case 5 : If $h\left(x_{i}\right)$ is changed from $h\left(x_{i}\right)<\varepsilon$ to $h\left(x_{i}\right)=\varepsilon$, the sample $x_{i}$ is changed from set $R$ to set $S$.

When set $S$ changes, we need to readjust the parameters of formula (5).

\section{Modeling and Updating Process}

1) determine the input and output variables of the model.

2) choose appropriate modeling parameters: penalty coefficient $C$, insensitivity coefficient $\varepsilon$ and kernel function, and establish the initial model based on support vector machine.

3) use the established model to estimate and predict output.

4) calculate the prediction deviation $e(k)$ between the actual output and the measured value in every sampling period.

5) determine whether the current time prediction error exceeds the maximum tolerable maximum error $E R R$. If $|e(k)|<E R R$, the model does not need to be updated and the model parameters remain unchanged. If $|e(k)| \geq E R R$, the model needs to be corrected. the input and output vector is added as the new sample to join the training set and the original sample set is readjusted.

6) If it need to end the identification process, quit, otherwise, go to step 3), repeat the whole calculation process.

Through the continuous replacement of the above steps, the samples are constantly updated, so that the model is constantly corrected, and online correction is achieved, with adaptive modeling [5].

\section{Simulation Experiment}

Online support vector machine is applied to model the dynamic process of turbine-boiler coordinated system. From the field running historical data, 600 groups of data are selected. The first step is data preprocessing, including high- pass filtering, low-pass filtering and coarse value processing. The parameter setting of the online support vector machine is as follows: the penalty coefficient $C=10$, the insensitive coefficient epsilon $\varepsilon=0.001$, the kernel function as the radial basis kernel function (RBF), the RBF kernel function provides a great flexibility for the nonlinear mapping of the input data, and is more effective for solving the complex nonlinear and inseparable classification problems.

The input of the load model is

$$
K\left(x_{i}, x_{j}\right)=\exp \left(\frac{-\left\|x_{i}-x_{j}\right\|}{2 \lambda^{2}}\right)
$$

$\left[\mu_{T}(t-1), \mu_{T}(t-2), \mu_{B}(t-1), \mu_{B}(t-2), N_{E}(t-1)\right]$

The input of main steam pressure model is

$\left[\mu_{T}(t-1), \mu_{T}(t-2), \mu_{B}(t-1), \mu_{B}(t-2), P_{T}(t-1)\right]$

The output of the load model is $N_{E}(t)$, and the output of main steam pressure model is $P_{T}(t)$.

Load training results are shown in Figure 1, the main steam pressure training results are shown in Figure 2. The load and the main steam pressure of the training results and the actual value is basically the same. 

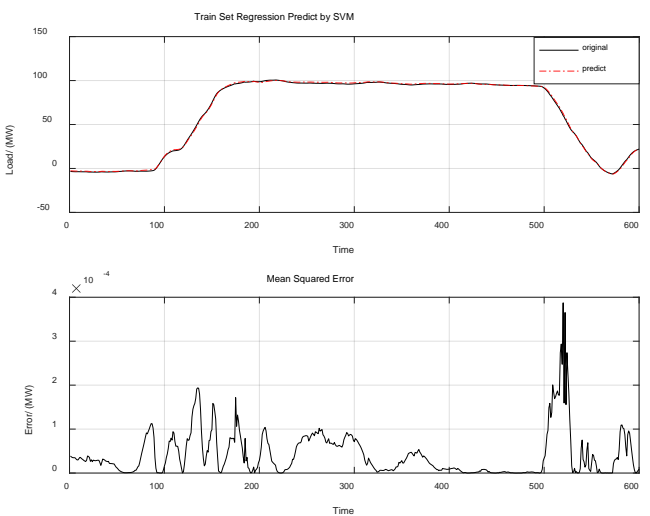

FIGURE 1. Training results of load model
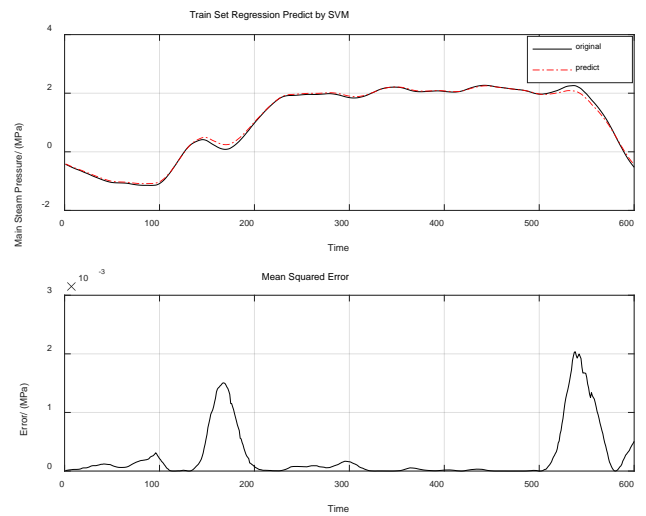

FIGURE 2. Training results of main steam pressure model

Based on the above built dynamic model of the boiler turbine coordination system, this section will further verify the accuracy and adaptability of the dynamic model. In order to verify the dynamic performance of the model, we select 2000 sets of continuous changes of the historical data as the input of the dynamic model, then compare the deviation between the predicted value and the actual measured value, and verify whether the model can be automatically adjusted to be more new when the deviation is large.

From Figure 3, the change trend of load and main steam pressure is basically consistent with the actual data collected in the field. The load model is updated 18 times, the time required for each update is $2.1324 \mathrm{~s}$, the efficiency model is updated 12 times, and the average time required for each update is 1.3378s. It can fully meet the requirements of online updating of models in practical applications, and use this model to achieve output prediction for future multiple times.
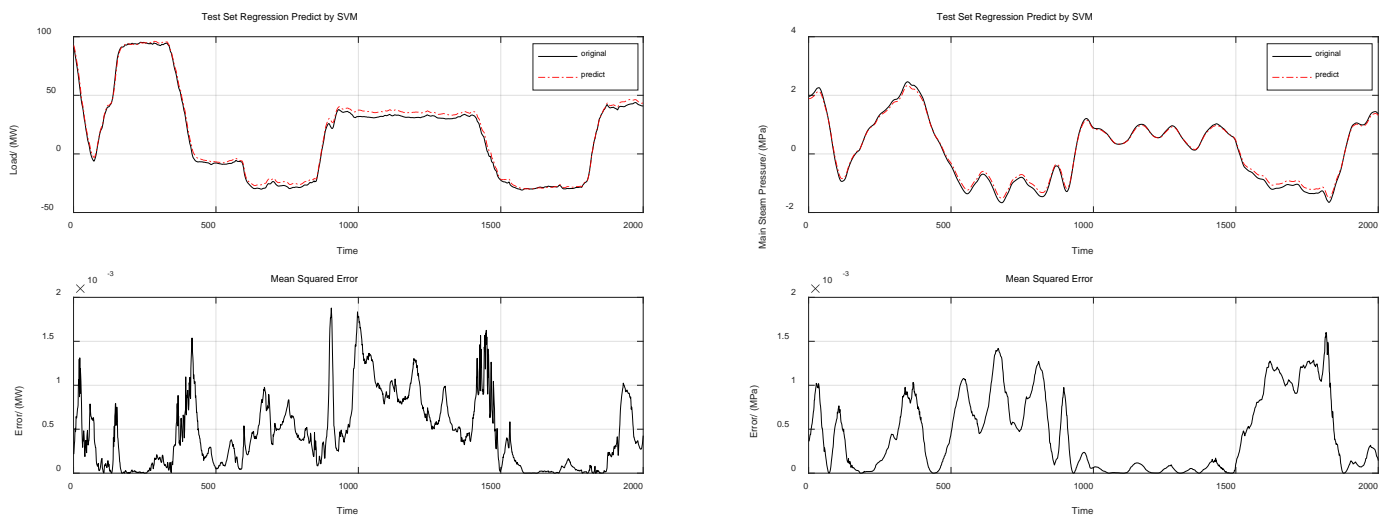

FIGURE 3. Comparison of model predicted value and actual value

\section{CONCLUSION}

In this paper, the dynamic modeling method of the coordination system based on online adaptive support vector machine is studied, and a mathematical model is established to reflect the characteristics of the coordination system, and the accuracy and adaptive ability of the model are verified by using the $600 \mathrm{MW}$ field operation data of a power plant. Compared with the steady state modeling method of support vector machine, the dynamic modeling method proposed in this paper can obviously improve the precision of the modeling of the coordination system. At the same time, it has the ability of using DCS data to correct the model of the coordinated system online, and can effectively deal with the influence of the non measurable disturbance such as coal quality. It provides a basis for the study of coordinated control. 


\section{ACKNOWLEDGMENTS}

This work was supported by the Fundamental Research Funds for the Central Universities (2018ZD05).

\section{REFERENCES}

1. Suykens J A K, Vandewalle J. Least Squares Support Vector Machine Classifiers[M]. Kluwer Academic Publishers, 1999.

2. Zheng J, Yu H, Shen F, et al. An Online Incremental Learning Support Vector Machine for Large-scale Data[C]. International Conference on Artificial Neural Networks. Springer Berlin Heidelberg, 2010:76-81.

3. Wang J G, Cheng L W, Zhang W X, et al. A Modified Incremental Support Vector Machine for Regression[J]. Applied Mechanics \& Materials, 2012, 135-136:63-69.

4. Wang W, Men C, Lu W. Online prediction model based on support vector machine[J]. Neurocomputing, 2008, 71(4-6):550-558.

5. Si F, Romero C E, Yao Z, et al. A new approach for function approximation in boiler combustion optimization based on modified structural AOSVR[J]. Expert Systems with Applications, 2009, 36(4):8691-8704. 